06

\title{
Влияние диоксида кремния на стабильность фазового состава и механические свойства керамики на основе диоксида циркония, упрочненной оксидом алюминия
}

\author{
(C) А.А. Дмитриевский, А.О. Жигачев, Д.Г. Жигачева, В.В. Родаев
}

Тамбовский государственный университет им. Г.Р. Державина, 392000 Тамбов, Россия

e-mail: aadmitr@yandex.ru

Поступило в Редакцию 13 марта 2020 г.

В окончательной редакции 15 апреля 2020 г.

Принято к публикации 24 апреля 2020 г.

\begin{abstract}
Исследовано влияние концентрации примеси $\mathrm{SiO}_{2}$ (в интервале от 0 до $10 \mathrm{~mol} \%$ ) на стабильность тетрагональной фазы $t-\mathrm{ZrO}_{2}$ и комплекс механических характеристик (на микро- и макроуровне) ATZкерамики на основе диоксида циркония, стабилизированного оксидом кальция $\left(C_{\mathrm{CaO}}=6.5 \mathrm{~mol} . \%\right)$, упрочненной оксидом алюминия $\left(C_{\mathrm{Al}_{2} \mathrm{O}_{3}}=5.8 \mathrm{~mol} \%\right.$ ). Установлено, что введение примеси $\mathrm{SiO}_{2}$ (в концентрации $C_{\mathrm{SiO}_{2}}=5 \mathrm{~mol} . \%$ ) приводит к почти двукратному увеличению вязкости разрушения (от $K_{C}=7.05 \mathrm{MPa} \cdot \mathrm{m}^{1 / 2}$ до $K_{C}=12.43 \mathrm{MPa} \cdot \mathrm{m}^{1 / 2}$ ) при незначительном уменьшении твердости (от $H=12.75$ до $10.9 \mathrm{GPa}$ ), а также к повышению предела прочности на сжатие (от $\sigma_{S}=2.44$ до $2.73 \mathrm{GPa}$ ) и пластичности (относительная деформация при сжатии увеличивается от $\varepsilon=5.3$ до 7.3\%) исследуемой ATZ-керамики. Показано, что указанные показатели достигнуты за счет снижения стабильности тетрагональной фазы $t-\mathrm{ZrO}_{2}$.
\end{abstract}

Ключевые слова: циркониевая керамика, оксид алюминия, диоксид кремния, структура, фазовый состав, механические свойства.

DOI: $10.21883 /$ JTF.2020.12.50128.84-20

\section{Введение}

Вплоть до 1975 г. чистая керамика на основе диоксида циркония находила свое применение лишь в качестве тугоплавкого (огнеупорного) материала. Ограничение для использования чистого $\mathrm{ZrO}_{2}$ в качестве инженерной или конструкционной керамики было обусловлено спонтанным фазовым переходом тетрагональной фазы $t-\mathrm{ZrO}_{2}$ в моноклинную $m-\mathrm{ZrO}_{2}$ при охлаждении $\left(\sim 950^{\circ} \mathrm{C}\right)$, сопровождающимся увеличением объема на $\sim 4 \%$, сдвиговой деформацией $\sim 16 \%$ и, как результат, разрушением материала [1]. Резкое расширение области практических приложений диоксидциркониевой керамики произошло благодаря открытию трансформационного упрочнения [2], обусловленного сохранением фазы $t-\mathrm{ZrO}_{2}$ при комнатной температуре путем введения стабилизаторов $\left(\mathrm{Y}_{2} \mathrm{O}_{3}, \mathrm{CeO}_{2}, \mathrm{MgO}, \mathrm{CaO}\right.$ и др.), приводящего к значительному улучшению механических свойств. На сегодняшний день стабилизированная диоксидциркониевая керамика обладает уникальным комплексом механических свойств: рекордной для оксидных керамик вязкостью разрушения $K_{C}$, высокими значениями износостойкости и прочности на изгиб, а также низким коэффициентом трения. С учетом низкой теплопроводности и высокой температуры плавления, химической инертности и радиационной стойкости это обеспечивает ей широчайшую область практических приложений от машиностроения до медицины $[3,4]$.
Одним из возможных и наиболее действенных способов дальнейшего улучшения рабочих характеристик керамики на основе диоксида циркония (в том числе соотношения твердость/трещиностойкость) является создание на ее основе композитов. Наиболее значительные успехи были достигнуты при комбинировании диоксида циркония и оксида алюминия [3-5]. В композитах $\mathrm{ZrO}_{2}+\mathrm{Al}_{2} \mathrm{O}_{3}$ сильные стороны диоксидциркониевой керамики сочетаются с достоинствами корундовой керамики (высокими значениями твердости $H$, модуля Юнга $E$ и прочности на сжатие $\left.\sigma_{C}\right)$. В общем случае в зависимости от соотношения концентраций $\mathrm{ZrO}_{2}$ и $\mathrm{Al}_{2} \mathrm{O}_{3}$ в композите различают корундовую керамику, упрочненную диоксидом циркония (Zirconia-Toughened Alumina или ZTA), и керамику на основе диоксида циркония, упрочненную оксидом алюминия (AluminaToughened Zirconia или ATZ) [6-9]. В последнем случае достижению высоких показателей механических свойств способствует одновременное проявление нескольких механизмов упрочнения: трансформационного, дисперсионного и механизма переноса нагрузки (load transfer). Трансформационный механизм упрочнения, присущий диоксиду циркония [1], обусловлен фазовым переходом тетрагональной фазы $t-\mathrm{ZrO}_{2}$ (метастабильной при комнатной температуре) в термодинамически устойчивую моноклинную фазу $m-\mathrm{ZrO}_{2}$. Такой переход сопровождается изменением удельного объема указанных фаз и возникновением сжимающих механических напряжений, тормозящих распространение трещин. В основе дис- 


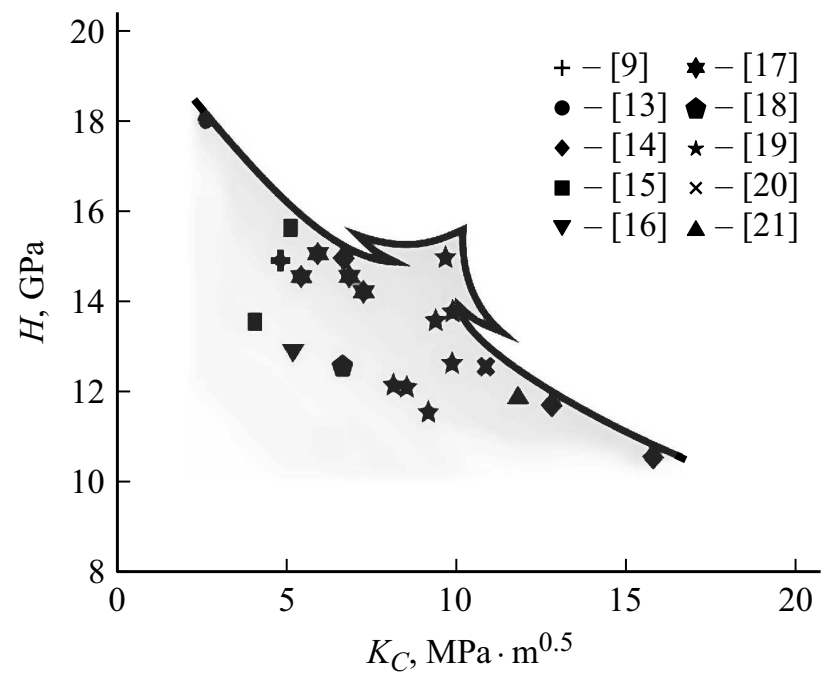

Рис. 1. Соотношение твердости Н и вязкости разрушения $K_{C}$ композиционных керамик $\mathrm{ZrO}_{2}+\mathrm{Al}_{2} \mathrm{O}_{3}$, отличающихся концентрациями компонент и условиями синтеза.

персионного механизма упрочнения лежит диссипация энергии распространяющейся трещины в результате ее отклонения от начального направления при „столкновении“ с более твердыми включениями (частицами) инородного материала $[10,11]$. Механизм переноса нагрузки проявляется при наличии протяженных армирующих элементов структуры, которые соединяют берега зарождающейся трещины и препятствуют их дальнейшему расхождению [12].

В зависимости от условий эксплуатации конкретного изделия более высокие требования могут предъявляться к той или иной характеристике композита. Целенаправленное управление свойствами (соотношением характеристик) композиционной керамики $\mathrm{ZrO}_{2}+\mathrm{Al}_{2} \mathrm{O}_{3}$ возможно благодаря вариативности ее состава и структуры. К наиболее важным факторам, во многом определяющим свойства композитов, относятся: процентное соотношение компонентов композита и характер их пространственного распределения, тип и концентрация стабилизатора (комбинации стабилизаторов) диоксида циркония, фазовый состав $\mathrm{ZrO}_{2}$, размер зерен $\mathrm{ZrO}_{2}$ и $\mathrm{Al}_{2} \mathrm{O}_{3}$, и пр. Варьирование перечисленных факторов позволяет управлять соотношением значений твердости и вязкости разрушения, например, от $H=18 \mathrm{GPa}$ и $K_{C}=2.7 \mathrm{MPa} \cdot \mathrm{m}^{1 / 2}[13]$ до $H=10.4 \mathrm{GPa}$ и $K_{C}=16 \mathrm{MPa} \cdot \mathrm{m}^{1 / 2}$ [14]. Сегодня многочисленные исследования разработчиков направлены на получение композитов $\mathrm{ZrO}_{2}+\mathrm{Al}_{2} \mathrm{O}_{3}$ с соотношением параметров $Y$ и $K_{C}$, превышающим условно проведенную линию, ограничивающую сверху „семейство“ экспериментальных данных, полученных разными научными коллективами (рис. 1) [9,13-21]. При этом разрабатываемые композиты должны обладать высокими значениями износостойкости, прочности на сжатие и на изгиб, стойкостью к гидротермальным воздействиям и пр.
Улучшению отдельных механических характеристик также может способствовать введение в композиционную керамику третьего компонента (допанта). Введение допанта может способствовать уменьшению среднего размера и разброса значений размера зерна керамических компонентов, препятствовать образованию микротрещин, связанному с различием температурных коэффициентов линейного расширения основных компонентов композита и пр. [21-23]. Так, в [21] было показано, что введение диоксида кремния в керамику на основе диоксида циркония (стабилизированную оксидом кальция) способствует повышению не только ее вязкости разрушения $\left(K_{C}=7.5-12.5 \mathrm{MPa} \cdot \mathrm{m}^{1 / 2}\right.$ при концентрации $C_{\mathrm{SiO}_{2}}=2.5 \mathrm{~mol} \%$ ), но и твердости $(\mathrm{H}=12-13 \mathrm{GPa}$ при $\left.C_{\mathrm{SiO}_{2}}=1 \mathrm{~mol} . \%\right)$. Это дает основание предполагать, что введение $\mathrm{SiO}_{2}$ в ATZ-керамику может положительно отразиться на ее свойствах. В связи с этим настоящая работа была направлена на исследование влияния диоксида кремния на стабильность фазового состава $\mathrm{ZrO}_{2}$ и комплекс механических характеристик диоксидциркониевой керамики (стабилизированной оксидом кальция), упрочненной оксидом алюминия.

\section{1. Экспериментальная методика}

Образцы ATZ-керамики изготавливали с использованием порошков $\mathrm{ZrO}_{2}$ (Sigma-Aldrich), $\mathrm{Al}_{2} \mathrm{O}_{3}$ (Hongwu), $\mathrm{CaO}$ (Reachem) и $\mathrm{SiO}_{2}$ (Sigma-Aldrich). Концентрацию стабилизатора $(\mathrm{CaO})$ по отношению к $\mathrm{ZrO}_{2}$ сохраняли неизменной $C_{\mathrm{CaO}}=6.5 \mathrm{~mol} \%$ и к полученной смеси добавляли $5.8 \mathrm{~mol} \% \mathrm{Al}_{2} \mathrm{O}_{3}$, что, согласно данным [18], обеспечивает высокое соотношение твердости и вязкости разрушения ATZ-керамики. Концентрацию диоксида кремния по отношению к смеси $\mathrm{CaO}+\mathrm{ZrO}_{2}+\mathrm{Al}_{2} \mathrm{O}_{3}$ варьировали в диапазоне от 0 до $10 \mathrm{~mol} . \%$.

Полученные смеси порошков диспергировали в дистиллированной воде (в массовом соотношении 1:3) при помощи ультразвука. Затем производили помол в планетарной мельнице Pulverisette (Fritsch) в течение $5 \mathrm{~h}$ шарами диаметром $1.5 \mathrm{~mm}$ при ускорении движения шаров $640 \mathrm{~m} / \mathrm{s}^{2}$. После помола следовала сушка смеси в печи при температуре $T_{0}=80^{\circ} \mid \mathrm{C}$ при нормальном давлении в течение $24 \mathrm{~h}$. Формовку образцов осуществляли путем одноосного сухого прессования при нагрузке $500 \mathrm{MPa}$ в течение $20 \mathrm{~min}$. Согласно [24,25], двухстадийный процесс спекания керамик на основе $\mathrm{ZrO}_{2}$ и $\mathrm{Al}_{2} \mathrm{O}_{3}$ обеспечивает сохранение более мелкого среднего размера зерна, чем при одностадийном спекании, и высокую плотность. В связи с этим спекание образцов в электрической печи проводили в двухстадийном режиме (детально описанном в [26]) с температурами $T_{1}=1300^{\circ} \mathrm{C}$ и $T_{2}=1200^{\circ} \mathrm{C}$.

Информацию о фазовом составе получали с использованием рентгеновского дифрактометра D2 Phaser (Bruker AXS) и сканирующего зондового комплекса микрорамановской спектроскопии NTEGRA SPECTRA 
(NT-MDT). Визуализацию структуры композиционной керамики и элементное картирование производили на сканирующем электронном микроскопе (СЭМ) высокого разрешения Merlin (Carl Zeiss).

Плотность изготовленных образцов определяли методом Архимеда при помощи взвешивания на лабораторных весах с пределом чувствительности $0.001 \mathrm{~g}$. Размер кристаллитов оценивали на основании данных об области когерентного рассеяния, вычисляемой из уширения дифракционных пиков по уравнению Шерера. При расчетах были использованы параметры кристаллических решеток из базы структур PDF 2 Международного центра дифракционных данных. Альтернативным методом определения среднего размера кристаллитов служил анализ СЭМ-изображений поверхности образцов. При этом измерения размеров кристаллитов производили в двух взаимно перпендикулярных направлениях. Усреднение осуществляли не менее чем по 250 отдельным кристаллитам.

Для исследования механических характеристик образцы ATZ-керамики подвергали механической шлифовке и полировке. Твердость по Виккерсу Н тестировали на автоматизированном микротвердомере Duramin A300 (EmcoTest) при нагрузках 50 N. $P-h$-диаграммы $(P-$ нагрузка на индентор, $h-$ глубина внедрения индентора) в том числе в режиме многоциклового нагружения (пирамида Берковича) регистрировали с использованием наноидентометра NanoIndenter G200 (MTS NanoInstruments). Важно отметить, что многоцикловое наноиндентирование с регистрацией $P-h$-диаграмм является одним из наиболее информативных методов диагностики механических свойств в области локальной деформации и сопутствующих фазовых превращений материала. Для определения модуля Юнга образцов также использовали наноидентометр NanoIndenter G200.

Для визуализации отпечатков индентора и измерения длин радиальных трещин использовали металлографический инвертированный микроскоп Aхіo Observer A1m (Carl Zeiss) с анализатором изображения Структура 5.0. Величину вязкости разрушения $K_{C}$ определяли, согласно [27], из выражения

$$
K_{C}=0.016\left(\frac{E}{H}\right)^{0.5} \frac{P}{l^{1.5}},
$$

где $E-$ модуль Юнга, $P$ - максимальная нагрузка на индентор $(50 \mathrm{~N}), l$ - длина радиальных трещин около отпечатка.

Прочность на сжатие определяли с использованием напольной двухколонной сервогидравлической испытательной машины MTS 870 Landmark (MTS). Для этого вырезали образцы в виде параллелепипедов с площадью поперечного сечения $2 \times 2 \mathrm{~mm}$.

Погрешности, указанные на графиках зависимостей, полученных экспериментальным путем, вычисляли как среднеквадратичные отклонения от средних значений.
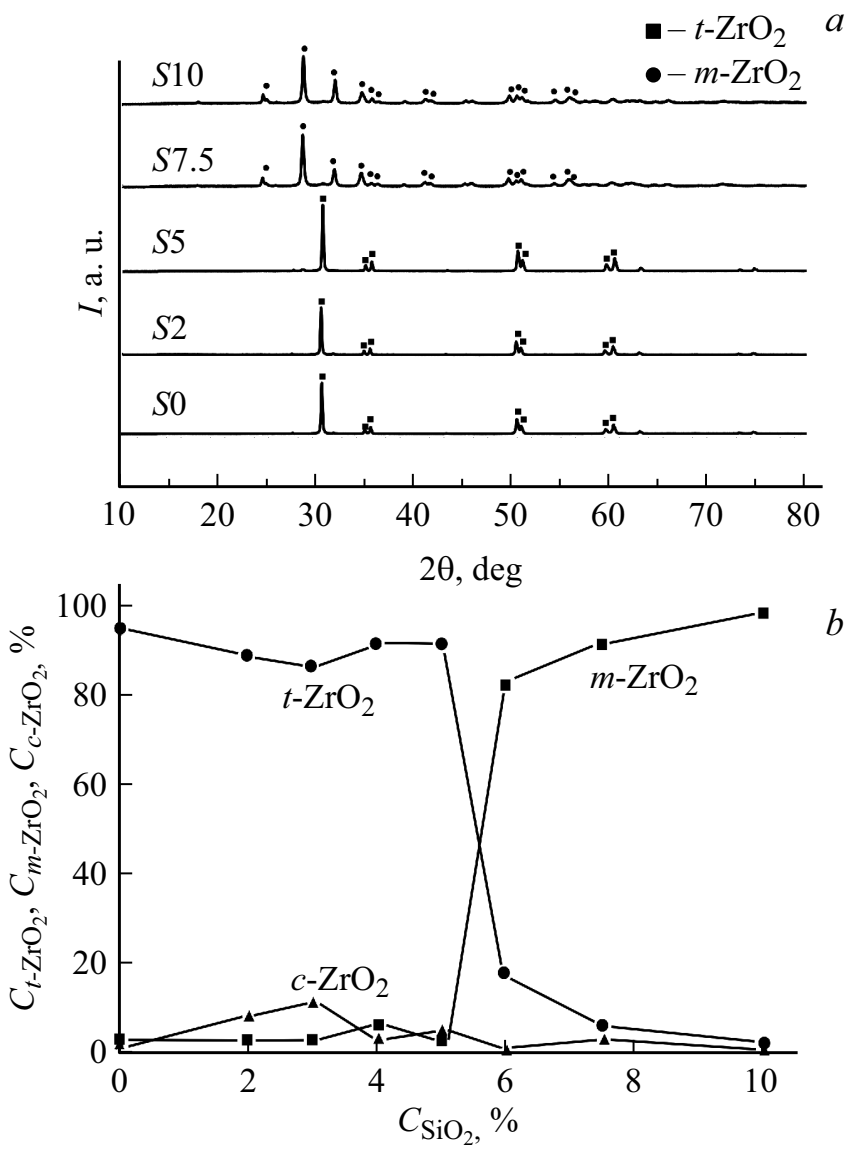

Рис. 2. Характерные дифрактограммы образцов ATZ-керамик с различным содержанием $\mathrm{SiO}_{2}(a)$ и зависимости относительных концентраций моноклинной, тетрагональной и кубической фаз диоксида циркония от концентрации $\mathrm{SiO}_{2}$ в ATZ-керамике $(b)$.

\section{2. Экспериментальные результаты}

На рис. 2, а представлено семейство характерных дифрактограмм, зарегистрированных на образцах ATZкерамики с различным содержанием $\mathrm{SiO}_{2}$. Для удобства дальнейшего описания образцы с содержанием $\mathrm{SiO}_{2}$ в концентрациях $0,2,5,7.5$ и $10 \mathrm{~mol} \%$ будем обозначать как $S 0, S 2, S 5, S 7.5$ и $S 10$ соответственно. Из представленных дифрактограмм видно, что образцы $S 0$, $S 2$ и $S 5$ характеризуются преимущественным содержанием тетрагональной фазы $t-\mathrm{ZrO}_{2}$, в то время как образцы $S 7.5$ и $S 10$ в основном содержат моноклинную фазу $m-\mathrm{ZrO}_{2}$. Анализ дифрактограмм с использованием процедуры Ритвельда (в программном обеспечении TOPAS) позволяет получать количественную информацию о фазовом составе образцов. На рис. $2, b$ представлены зависимости относительной концентрации моноклинной $\left(m-\mathrm{ZrO}_{2}\right)$, тетрагональной $\left(t-\mathrm{ZrO}_{2}\right)$ и кубической $\left(c-\mathrm{ZrO}_{2}\right)$ фаз исследуемых образцов от концентрации в них $\mathrm{SiO}_{2}$. Видно, что повышение содержания $\mathrm{SiO}_{2}$ (до $C_{\mathrm{SiO}_{2}}=5 \mathrm{~mol} \%$ ) в $\mathrm{ATZ}$-керамике не вызывает существенных изменений соотношения кон- 

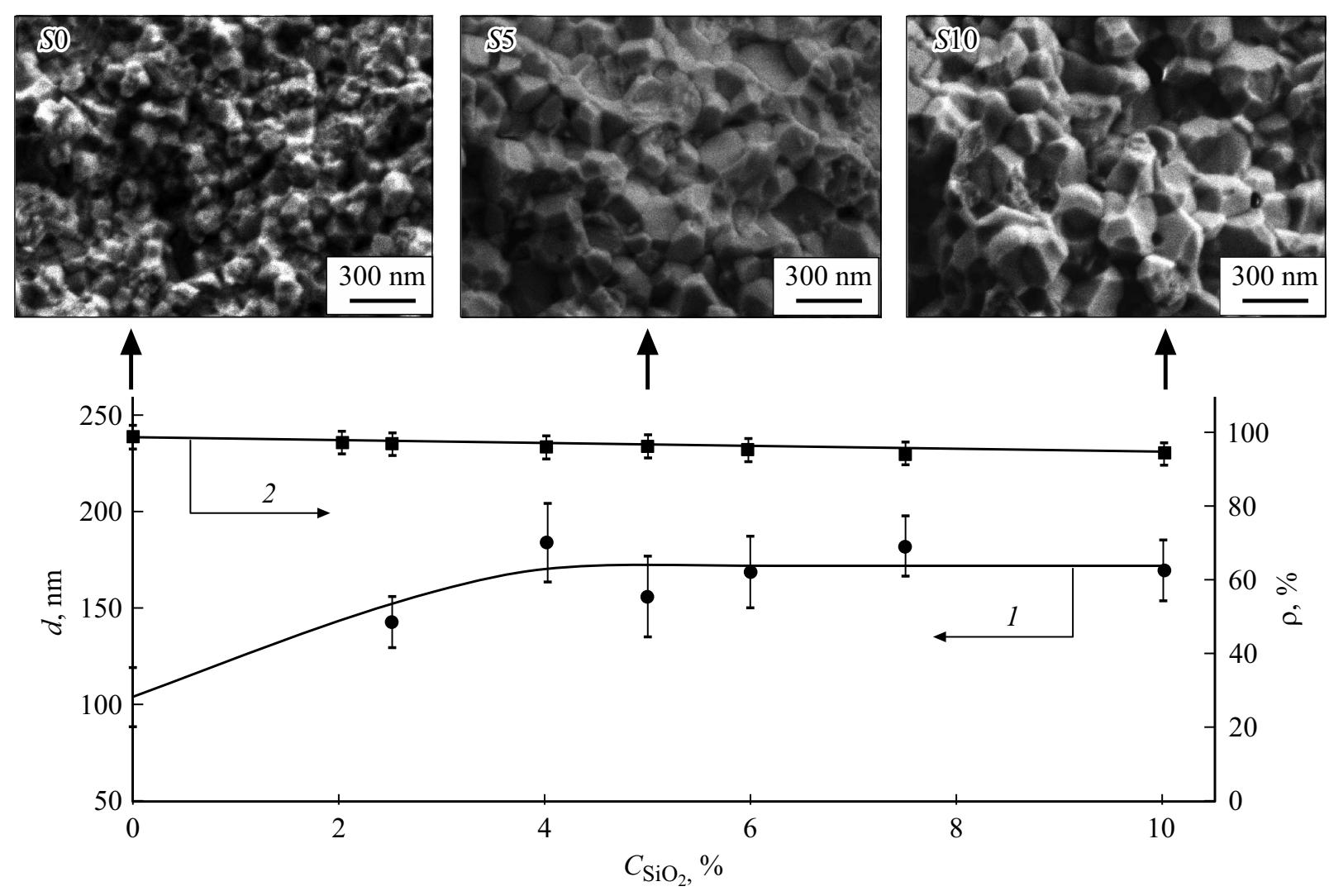

Рис. 3. Зависимости среднего размера зерна (1) и относительной плотности (2) ATZ-керамик от концентрации $\mathrm{SiO}_{2}$. На врезке представлены СЭМ-изображения участков поверхности скола образцов $S 0, S 5$ и $S 10$.

центраций моноклинной, тетрагональной и кубической фаз диоксида циркония. Доля тетрагональной фазы сохраняется на уровне $C_{t-\mathrm{ZrO}_{2}}=86-91 \%$. Однако в интервале концентраций $5 \mathrm{~mol} . \%<C_{\mathrm{SiO}_{2}}<6 \mathrm{~mol} \%$ н наблюдается резкое перераспределение фазового состава. При $C_{\mathrm{SiO}_{2}}>6 \mathrm{~mol} \%$ доминирующей фазой становится моноклинная. Ее относительная доля при $C_{\mathrm{SiO}_{2}}=10 \mathrm{~mol} . \%$ достигает 95\%. Доля кубической фазы диоксида циркония $C_{c-\mathrm{ZrO}_{2}}$ остается в среднем неизменной (на уровне единиц процентов) во всем исследованном интервале концентраций $\mathrm{SiO}_{2}$ в ATZ-керамике.

На рис. 3 сопоставлены зависимости среднего размера зерна $d_{\mathrm{ZrO}_{2}}$ и плотности $\rho \mathrm{ATZ}$-керамики от содержания в ней $\mathrm{SiO}_{2}$. Видно, что введение $\mathrm{SiO}_{2}$ в концентрации до $4 \mathrm{~mol} \%$ сопровождается увеличением $d_{\mathrm{ZrO}_{2}}$. В интервале концентраций $4 \mathrm{~mol} . \% \leq C_{\mathrm{SiO}_{2}} \leq 10 \mathrm{~mol} . \%$ эта зависимость выходит в насыщение. Изначально высокая плотность ATZ-керамики практически не зависит от содержания $\mathrm{SiO}_{2}$ (в исследованном интервале концентраций от 0 до $10 \mathrm{~mol} . \%)$ и с учетом погрешности измерений сохраняет значения на уровне 94.5-99\%. В качестве примера, иллюстрирующего описанные зависимости, на врезке к рис. 3 представлены СЭМизображения участков поверхности скола образцов $S 0$, $S 5$, и $S 10$.
Наблюдаемые изменения структуры (размера зерна) и фазового состава ATZ-керамики (при варьировании содержания $\mathrm{SiO}_{2}$ ) должны отражаться на ее механических свойствах. При этом наиболее существенных изменений следует ожидать в области 5-процентной концентрации $\mathrm{SiO}_{2}$.

Действительно, обнаружено уменьшение твердости (измеренной при индентировании пирамидой Виккерса) ATZ-керамики по мере повышения концентрации $\mathrm{SiO}_{2}$. Показано (рис. 4, $a$, кривая 1 ), что зависимость $H\left(C_{\mathrm{SiO}_{2}}\right)$ имеет 2 характерные области, отличающиеся углом наклона. В интервале $0 \mathrm{~mol} \%<C_{\mathrm{SiO}_{2}}<5 \mathrm{~mol} \%$ твердость уменьшается незначительно (от $12.7 \pm 0.25 \mathrm{GPa}$ до $10.9 \pm 0.5 \mathrm{GPa})$. Второй участок $\left(C_{\mathrm{SiO}_{2}}>5 \mathrm{~mol} . \%\right)$ характеризуется более существенной деградацией твердости. Так, при $C_{\mathrm{SiO}_{2}}=10 \mathrm{~mol} \%$ твердость падает до значений $4.3 \pm 0.35 \mathrm{GPa}$.

В противоположность этому, введение $\mathrm{SiO}_{2}$ в ATZкерамику в концентрациях $C_{\mathrm{SiO}_{2}} \leq 5 \mathrm{~mol} \%$ п положительно отражается на вязкости разрушения. При этом на фоне незначительного $(\sim 15 \%)$ снижения $H$ наблюдается весьма существенное (до 75\%) увеличение $K_{C}$ ATZ-керамики (рис. 4, $\mid a$, кривая 2). Так, при $C_{\mathrm{SiO}_{2}}=5 \mathrm{~mol} . \% K_{C}=12.43 \pm 0.3 \mathrm{MPa} \cdot \mathrm{m}^{1 / 2}$, в то время как при $C_{\mathrm{SiO}_{2}}=0 \mathrm{~mol} . \% K_{C}=7.05 \pm 0.35 \mathrm{MPa} \cdot \mathrm{m}^{1 / 2}$. Дальнейшее увеличение содержания $\mathrm{SiO}_{2}$ в ATZ- 

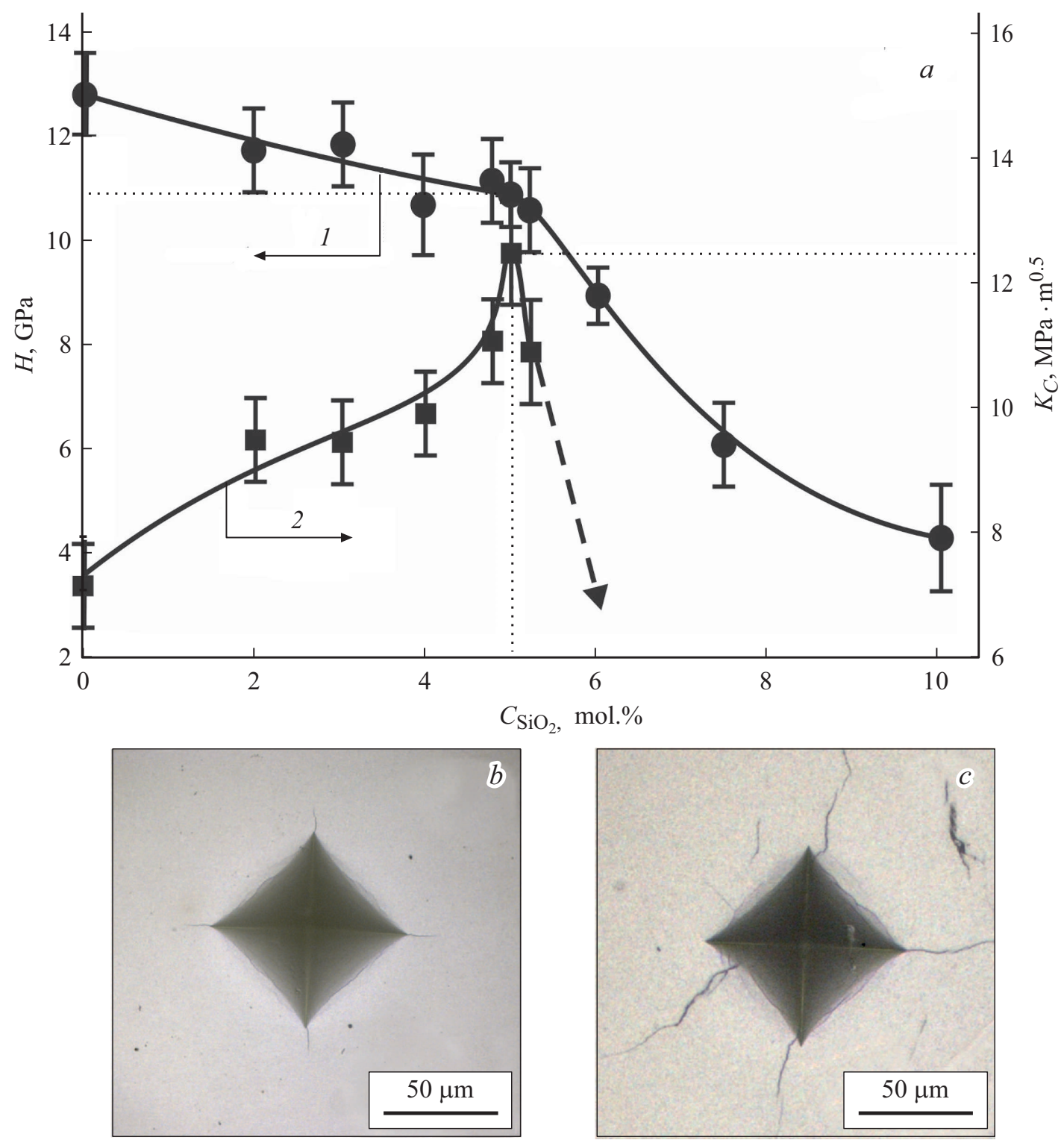

Pис. 4. Зависимости твердости и вязкости разрушения $\mathrm{ATZ}$-керамики от концентрации $\mathrm{SiO}_{2}(a)$ и микрофотографии отпечатков индентора, сформированных в образцах ATZ-керамики с содержанием диоксида кремния $C_{\mathrm{SiO}_{2}}=5(b)$ и 6 mol.\% $(c)$.

керамике $\left(C_{\mathrm{SiO}_{2}}>5.2 \mathrm{~mol} \%\right.$ \%) вызывает резкое падение вязкости разрушения. При этом материал в окрестности отпечатка индентора начинает разрушаться и трещины распространяются хаотично. В качестве примера на рис. 4, $b, c$ представлены микрофотографии отпечатков пирамиды Виккерса, сформированных в образцах с содержанием $\mathrm{SiO}_{2} 5$ и 6 mol.\% соответственно. Отсутствие четко выраженных радиальных трещин не позволяет определить значения вязкости разрушения при $C_{\mathrm{SiO}_{2}} \geq 6 \mathrm{~mol} \%$. Появление подобного семейства трещин (рис. 4,c) свидетельствует о резком охрупчивании ATZ-керамики при содержании в ней диоксида кремния более $6 \mathrm{~mol} . \%$.

Следует отметить, что максимального значения $K_{V C}$ достигает при той же концентрации $\mathrm{SiO}_{2}$, при которой наблюдается перелом зависимости $H\left(C_{\mathrm{SiO}_{2}}\right)$. Этот факт нетривиален и заслуживает внимания, поскольку обычно твердость и вязкость разрушения демонстрируют противоположные отклики на структурные изменения материала. С учетом того что при $C_{\mathrm{SiO}_{2}} \geq 4 \mathrm{~mol} \%$ средний размер зерна не изменяется (рис. 3 , кривая 1 ), наблюдаемая резкая деградация $H$ и $K_{C}$ при $C_{\mathrm{SiO}_{2}}>5 \mathrm{~mol} . \%$ не находит объяснения с позиции закона Холла-Петча. Другими словами, размер зерна, как и относительная плотность, ATZ-керамики не являются определяющими факторами в наблюдаемом явлении.

Таким образом, из всех исследованных образцов наибольший интерес вызывает ATZ-керамика, содержащая $5 \mathrm{~mol} \% \quad \mathrm{SiO}_{2}$, для которой характерно сочетание высокой твердости и вязкости разрушения 
$a$
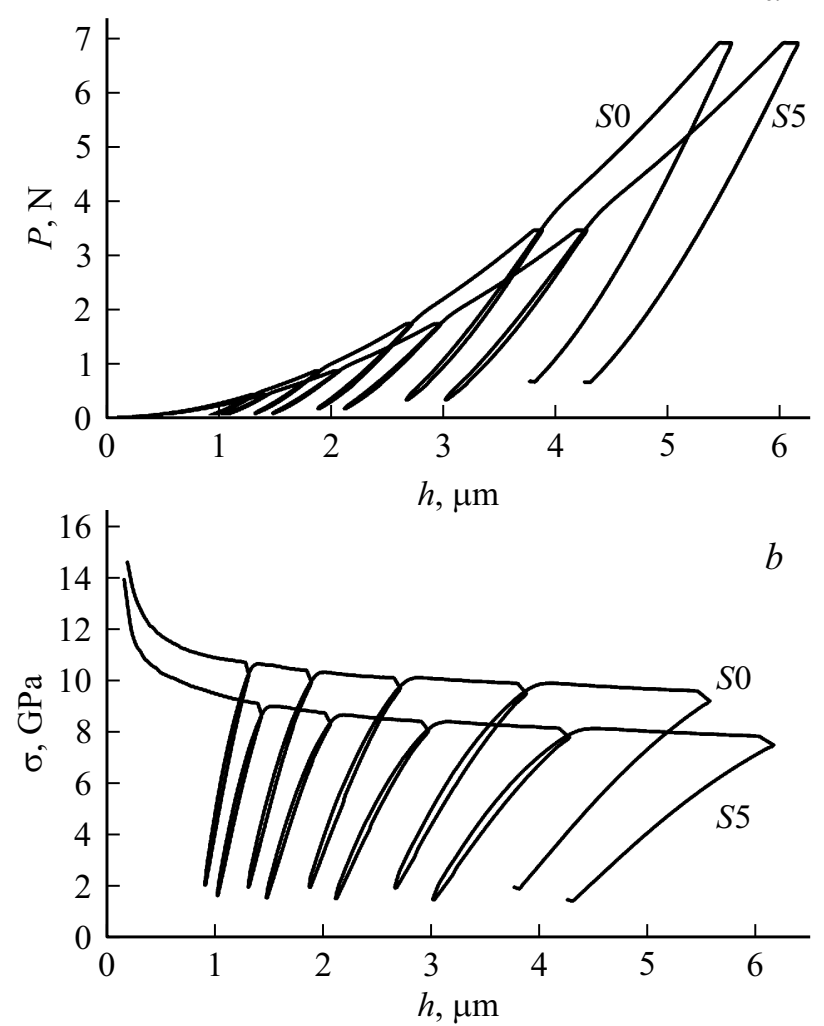

Рис. 5. Характерные $P-h$-диаграммы $(a)$ и зависимости средних контактных напряжений $(b)$ от глубины внедрения индентор $h$. Кривые 1 и 2 соответствуют образцам $S 0$ и $S 5$ соответственно.

$\left(H=10.9 \pm 0.5 \mathrm{GPa}, K_{C}=12.43 \pm 0.3 \mathrm{MPa} \cdot \mathrm{m}^{1 / 2}\right)$. В связи с этим дальнейшие более детальные исследования проводили на образцах ATZ-керамики с нулевым и 5-процентным содержанием $\mathrm{SiO}_{2}(\mathrm{~S} 0$ и $S 5$ соответственно).

На рис. 5, $a$ сопоставлены типичные $P-h$-диаграммы, зарегистрированные при индентировании (5 циклов нагружения-разгрузки с последовательным нарастанием $P$ ) образцов $S 0$ и $S 5$. Видно, что наклон плеча нагрузки $P-h$-диаграмм у образцов S5 более пологий по сравнению с образцами $S 0$. Это согласуется с результатами, полученными при индентировании пирамидой Виккерса под нагрузкой $P=50 \mathrm{~N}$ (рис. 4, $a$, кривая 1 ). Метод многоциклового индентирования позволяет наблюдать гистерезисные петли, образующиеся между плечом разгрузки одного из циклов и плечом нагрузки следующего цикла, в материалах, для которых характерны фазовые превращения при сосредоточенных нагрузках. На представленных $P$-h-диаграммах и зависимостях средних контактных напряжений под индентором от глубины его внедрения $h$ (рис. 5, $a, b$ ) такие петли хорошо заметны в обоих типах образцов ( $S 0$ и $S 5)$. По мере увеличения глубины внедрения индентора (от цикла к циклу) ширина гистерезисных етель увеличивается, что соответствует данным, полученным на разных материалах [28].
Сам факт формирования гистерезисной петли на $P-h$ диаграмме означает, что при повторном нагружении индентор внедряется в материал, структура которого отличается от исходной. Причиной такого структурного изменения, как правило, являются фазовые превращения [28]. В этом случае ширина гистерезисной петли характеризует долю новой фазы. В предположении, что причиной наблюдаемых (рис. 5) гистерезисных потерь является фазовое превращение $t-\mathrm{ZrO}_{2} \rightarrow m-\mathrm{ZrO}_{2}$, относительная доля материала, претерпевшего фазовый переход, пропорциональна величине $\delta$, рассчитываемой по аналогии с [29] из следующего выражения:

$$
\delta=\frac{W_{\text {ioop }}}{W_{P}} \cdot 100 \%,
$$

где $W_{\text {loop }}$ - энергия гистерезисных потерь в данном цикле, а $W_{P}$ - энергия пластической деформации. Значения $W_{\text {loop }}$ и $W_{P}$ могут быть определены путем численного интегрирования данных $P-h$-диаграмм. Расчет показывает, что для образцов $S 0$ и $S 5$ относительные доли новой (моноклинной) фазы пропорциональны значениям $\delta_{1}=1.97 \pm 0.05 \%$ и $\delta_{2}=2.16 \pm 0.05 \%$ соответственно. Отметим также, что введение в ATZкерамику $\mathrm{SiO}_{2}$ приводит к уменьшению средних контактных напряжений, достаточных для фазовых превращений $t-\mathrm{ZrO}_{2} \rightarrow m-\mathrm{ZrO}_{2}$ в процессе формирования отпечатка индентора от $\sigma_{C}=10.8-9.6 \mathrm{GPa}$ (в зависимости от номера цикла нагружения) до $\sigma_{C}=9.2-7.9 \mathrm{GPa}$ (рис. $5, b)$. То есть примесь диоксида кремния способствует снижению критических напряжений, вызывающих $t \rightarrow m$-переход, и, таким образом, повышает трансформируемость тетрагональной фазы $\left(t-\mathrm{ZrO}_{2} \rightarrow m-\mathrm{ZrO}_{2}\right)$.

Подтверждением этому выводу являются данные микрорамановской спектроскопии. На рис. 6, $a$ приведены в соответствие спектры рамановского смещения, зарегистрированные в области выхода радиальных трещин из углов отпечатков индентора (пирамида Виккерса), сформированных в образцах $S 0$ и $S 5$. Оба спектра характеризуются наличием сигналов, которые, согласно [3033], соответствуют моноклинной $m-\mathrm{ZrO}_{2}$ (линии 105 , $181,375,474 \mathrm{~cm}^{-1}$ ) и тетрагональной $t-\mathrm{ZrO}_{2}$ (линии 145 , 260,320 и $644 \mathrm{~cm}^{-1}$ ) фазам диоксида циркония. При этом видно, что введение $\mathrm{SiO}_{2}$ в ATZ-керамику приводит к снижению интенсивности линий $\left(145,260\right.$ и $\left.644 \mathrm{~cm}^{-1}\right)$, соответствующих $t-\mathrm{ZrO}_{2}$ и пропорциональному увеличению интенсивности линий $m-\mathrm{ZrO}_{2}$ (рис. $\left.6, a\right)$. Не смотря на то, что сравнительный анализ рамановских спектров позволяет лишь на качественном уровне оценивать изменение фазового состава материалов, представленные на рис. 6, $a$ данные свидетельствуют о меньшем содержании тетрагональной фазы $\mathrm{C} t-\mathrm{ZrO}_{2}$ в деформированной индентором области образца $S 5$ по сравнению с образцом S0. Другими словами, в ATZ-керамике с 5-процентным содержанием $\mathrm{SiO}_{2}$ процесс фазовых превращений $t-\mathrm{ZrO}_{2} \rightarrow m-\mathrm{ZrO}_{2}$ под индентором происходит эффективнее, чем в ATZ-керамике, не содержащей $\mathrm{SiO}_{2}$. 

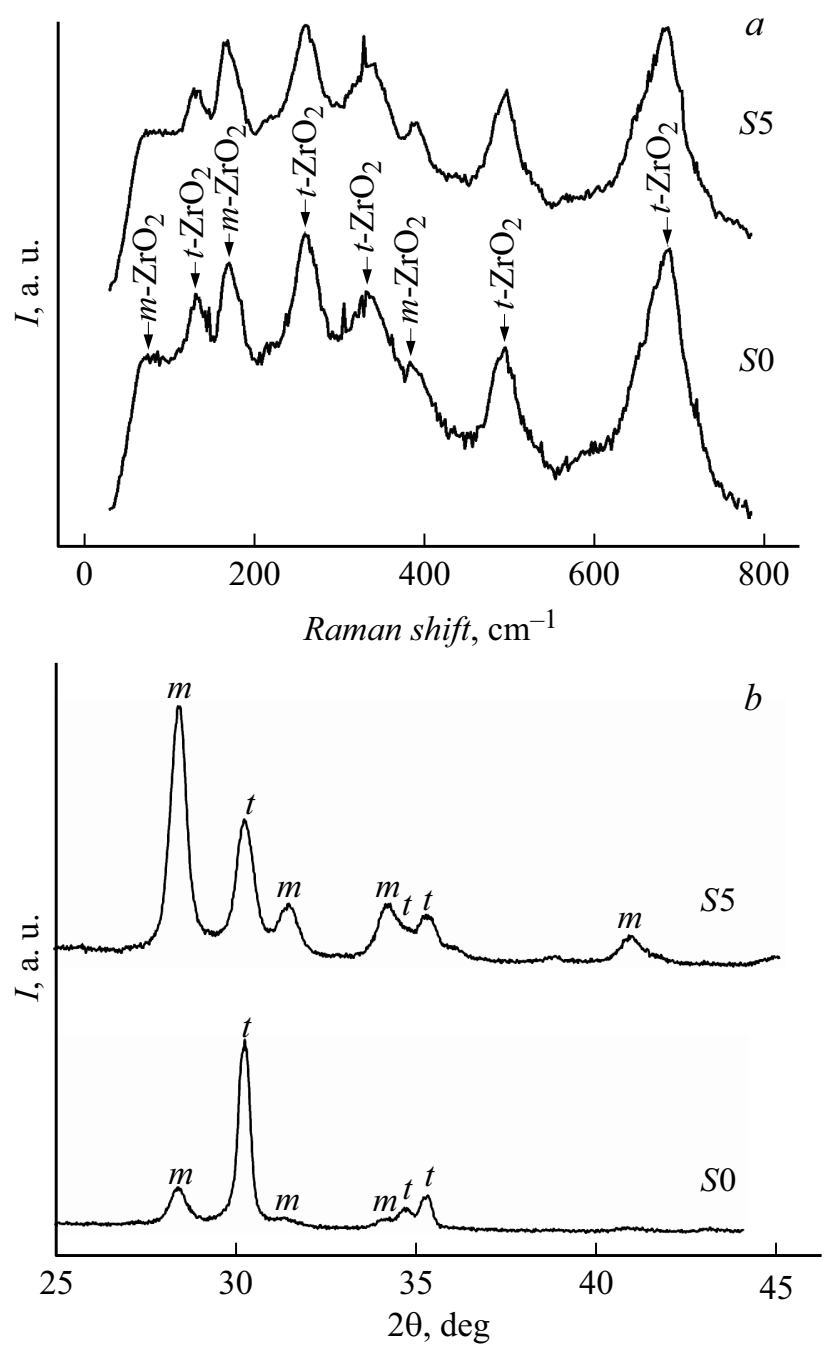

Рис. 6. Типичные спектры рамановского смещения, зарегистрированные в областях выхода радиальных трещин из углов отпечатков индентора $(a)$, и характерные дифракционные картины $(b)$, полученные на образцах ATZ-керамики с нулевым $(S 0)$ и 5-процентным $(S 5)$ содержанием $\mathrm{SiO}_{2}$.

Наконец, сравнение дифракционных картин, зарегистрированных на сколах образцов $S 0$ и $S 5$ (рис. 6,b), демонстрирует существенное различие их фазового состава. Видно, что введение $\mathrm{SiO}_{2}$ в ATZ-керамику приводит к значительному росту интенсивности сигналов от моноклинной фазы $m-\mathrm{ZrO}_{2}$ (линии 28.5, 31.5, 34.2 и 410) и пропорциональному уменьшению интенсивности сигналов от тетрагональной фазы $t-\mathrm{ZrO}_{2}$ (линии 30.2, 34.7, 35.30). Анализ дифрактограмм с использованием процедуры Ритвельда позволил произвести количественную оценку фазового состава образцов $S 0$ и $S 5$ в местах скола. Установлено, что моноклинная, тетрагональная и кубическая фазы $\mathrm{ZrO}_{2}$ в области скола образца $S 0$ находятся в соотношении 27:68:5\% соответственно, в то время как образец $S 5$ имеет соотношение указанных фаз 74:22:4\%. Это распространяет сделанный выше вывод об увеличении эффективности фазовых превращений
$t-\mathrm{ZrO}_{2} \rightarrow m-\mathrm{ZrO}_{2}$ при введении $\mathrm{SiO}_{2}$ в ATZ-керамику на уровень макропластической деформации.

Таким образом, результаты, полученные различными методами, показывают, что добавление $5 \mathrm{~mol} . \% \mathrm{SiO}_{2}$ в исследуемую ATZ-керамику повышает трансформируемость тетрагональной фазы $t-\mathrm{ZrO}_{2}$.

На рис. 7 представлены СЭМ-изображения, иллюстрирующие отпечаток индентора с радиальными трещинами в образце $S 5$ и отклонения трещин при „столкновении“ с зернами $\mathrm{Al}_{2} \mathrm{O}_{3}$ (более темные области, согласно $[7,10,11])$. Отклонения сопровождаются диссипацией упругой энергии и, как следствие, приводят к увеличению вязкости разрушения материала. Такое поведение однозначно указывает на то, что разработанные материалы являются композитами, то есть $\mathrm{Al}_{2} \mathrm{O}_{3}$ действует в диоксидциркониевой керамике как упрочняющая добавка $[10,11,27,34,35]$.

В отдельной серии измерений было показано, что добавление диоксида кремния $\left(C_{\mathrm{SiO}_{2}}=5 \mathrm{~mol} . \%\right)$ положительно сказывается и на макромеханических характеристиках ATZ-керамики. Для этого специально изготовленные в виде плоскопараллельных брусков образцы типа $S 0$ и $S 5$ (по 8 штук) деформировали трехточечным изгибом до разрушения. Установлено, что образцы $S 5$ более пластичны и имеют более высокую прочность. Так, при введении $5 \mathrm{~mol} \% \mathrm{SiO}_{2}$ в ATZ-керамику ее относительная деформация при сжатии увеличилась от $\varepsilon=5.3 \pm 0.3 \%$ до $\varepsilon=7.3 \pm 0.5 \%$, а предел прочности на сжатие увеличился от $\sigma_{S}=2.44 \pm 0.1 \mathrm{GPa}$ до $\sigma_{S}=2.73 \pm 0.15 \mathrm{GPa}$.

\section{3. Обсуждение}

Введение диоксида кремния в керамику на основе диоксида циркония может оказывать существенное влияние на ее структуру. Прежде всего, $\mathrm{SiO}_{2}$, как известно, способствует спеканию диоксидциркониевой керамики [36]. Это может способствовать повышению плотности и улучшению механических свойств. Например, авторы [21] наблюдали снижение пористости и увеличение твердости диоксидциркониевой керамики (стабилизированной оксидом кальция) при введении в ее состав $\mathrm{SiO}_{2}$ в концентрации $C_{\mathrm{SiO}_{2}}=0.5-2.5 \mathrm{~mol} . \%$.

Из представленных на рис. 3 данных видно, что с увеличением содержания $\mathrm{SiO}_{2}$ (в интервале от 0 до 4 mol.\%) увеличивается средний размер зерна $d_{\mathrm{ZrO}_{2}}$. Это указывает на то, что $\mathrm{SiO}_{2}$ действует как спекающая добавка для ATZ-керамики, что в свою очередь согласуется с данными [36].

С другой стороны, как было показано, например, в [23,37], добавление диоксида кремния в композит, содержащий в качестве стабилизатора $\mathrm{CaO}$, может сопровождаться образованием силикатов кальция. Это эквивалентно уменьшению относительной доли стабилизатора. В свою очередь, уменышение концентрации стабилизатора $C_{\mathrm{CaO}}$ (относительно $\mathrm{ZrO}_{2}$ ) может вести 

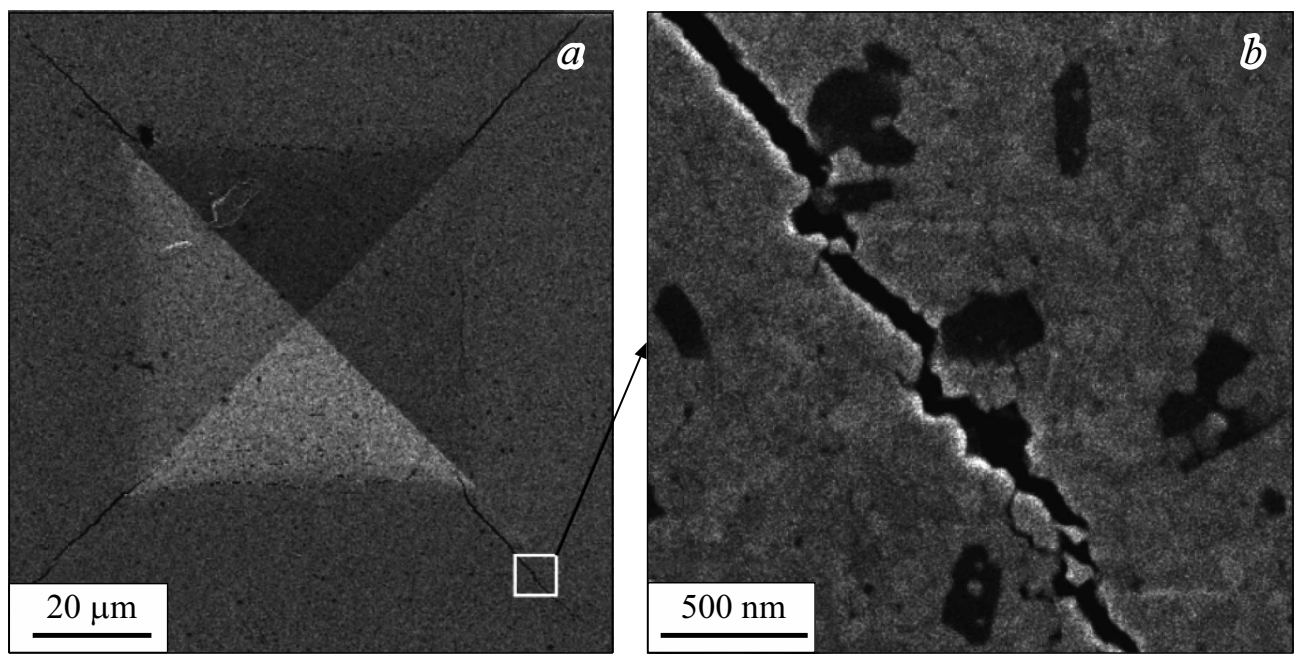

Рис. 7. СЭМ-изображения отпечатка индентора с радиальными трещинами $(a)$ и участка радиальной трещины $(b)$ в образце $S 5$.

к спонтанному $t \rightarrow m$-превращению при охлаждении после спекания и, как следствие, к ухудшению механических свойств керамики. Кроме того, в исследуемой системе $\mathrm{CaO}-\mathrm{ZrO}_{2}+\mathrm{Al}_{2} \mathrm{O}_{3}+\mathrm{SiO}_{2}$ возможно образование алюмосиликатов. Однако образование алюмосиликатов не может служить объяснением наблюдаемого изменения фазового состава диоксида циркония (рис. 2), поскольку $\mathrm{Al}_{2} \mathrm{O}_{3}$ является упрочняющей добавкой (за счет дисперсионного механизма упрочнения), а не стабилизатором (рис. 7).

Появление силикатов в кристаллической фазе должно отражаться на регистрируемых дифрактограммах. Представленные на рис. 2 дифрактограммы образцов $S 0-S 10$ не содержат пики, соответствующие диоксиду кремния или силикатам. Это означает, что исследуемая ATZкерамика не содержит силикатов в кристаллической фазе. Несмотря на отсутствие соответствующих пиков, остается очевидным, что диоксид кремния взаимодействует с оксидом кальция, снижая тем самым стабильность тетрагональной фазы $t-\mathrm{ZrO}_{2}$. Это следует из резкого падения вязкости разрушения при $C_{\mathrm{SiO}_{2}}>5 \mathrm{~mol} . \%$, a также резкого увеличения доли моноклинной фазы $m-\mathrm{ZrO}_{2}$ в том же диапазоне концентраций $\mathrm{SiO}_{2}$. Таким образом, кремнийсодержащие фазы (силикаты) находятся в ATZ-керамике в аморфном состоянии. Остается вопрос, сконцентрирована ли эта фаза в тройных стыках зерен, или равномерно распределена по границам зерен. Анализ СЭМ-изображений не позволил выявить какие-либо отдельные участки, которые можно было бы отождествить с фазами, обогащенными $\mathrm{SiO}_{2}$.

Дополнительно было проведено элементное картирование (методом EDS) участка поверхности скола образца S5. На рис. 8 приведены в соответствие СЭМизображение такого участка (а) и карты распределения $\mathrm{Zr}(b), \mathrm{Al}(c)$ и $\mathrm{Si}(d)$ по тому же участку поверхности. На рис. $8, b$, с отчетливо видны неоднородности в распределении элементов, которые связаны с наличием зерен $\mathrm{Al}_{2} \mathrm{O}_{3}$ в матрице из $\mathrm{ZrO}_{2}$. Диоксид кремния распределен гораздо более однородно (рис. $8, d$ ). Незначительные, хотя и заметные, вариации, вероятно, связаны с топологией. Это позволяет предположить, что обогащенная диоксидом кремния фаза равномерно распределена в материале. Наиболее вероятно эта фаза распределена в виде тонкого аморфного слоя на границах зерен, поскольку $\mathrm{SiO}_{2}$ и соответствующие силикаты не растворяются в диоксиде циркония при комнатной температуре. Аналогичный вывод был сделан авторами [21] при исследовании влияния $\mathrm{SiO}_{2}$ на фазовый состав и механические свойства диоксидциркониевой керамики, стабилизированной оксидом кальция. Кроме того, о подобном распределении обогащенной диоксидом кремния фазы сообщалось ранее [23] при исследовании керамики на основе диоксида циркония биомедицинского назначения. Авторами [23] было показано, что наличие $\mathrm{SiO}_{2}$ способствует закруглению углов зерен и может повышать долговременную стабильность тетрагональной фазы $t-\mathrm{ZrO}_{2}$.

Отметим, что как было показано выше, введение $\mathrm{SiO}_{2}$ в ATZ-керамику, стабилизированную $\mathrm{CaO}$, приводит к снижению энергетического порога для $t \rightarrow m$-превращений в области распространяющейся трещины, а не к повышению стабильности тетрагональной фазы $t-\mathrm{ZrO}_{2}$, которое наблюдалось в диоксидциркониевой керамике, стабилизированной $\mathrm{Y}_{2} \mathrm{O}_{3}$ [23]. Кроме того, в отличие от [21 и 23], где улучшение механических свойств и стойкости к низкотемпературной деградации авторы наблюдали при концентрации $\mathrm{SiO}_{2} 1.5 \pm 2.5 \mathrm{~mol} \%$ и $0.5 \mathrm{~mol} . \%$ соответственно, обнаруженное нами увеличение вязкости разрушения ATZкерамики достигается при $C_{\mathrm{SiO}_{2}}=5 \mathrm{~mol} . \%$. Столь существенные различия в оптимальном содержании $\mathrm{SiO}_{2}$ могут быть обусловлены, во-первых, присутствием в ATZ-керамике примеси $\mathrm{Al}_{2} \mathrm{O}_{3}$ и соответственно „потерей“ части $\mathrm{SiO}_{2}$ на образование силикатов алюминия, 


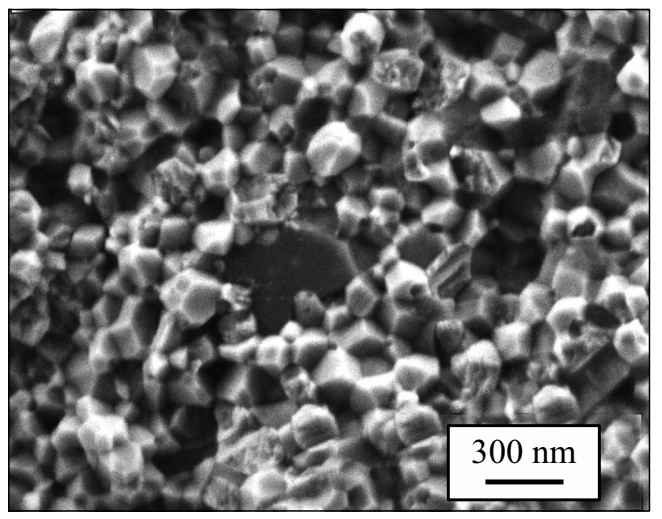

$c$

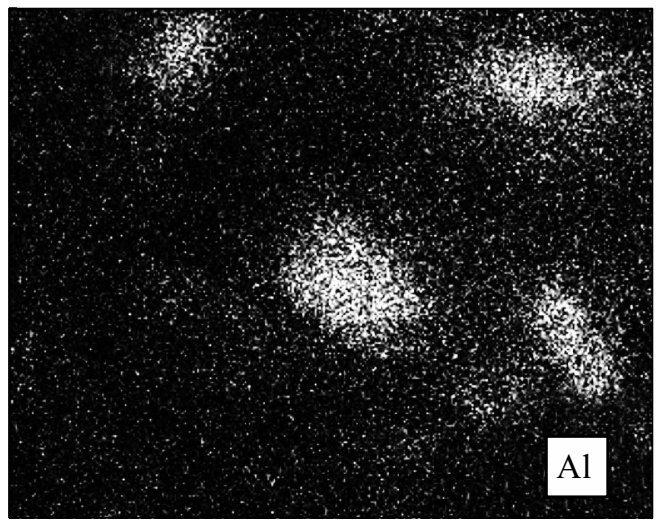

$b$

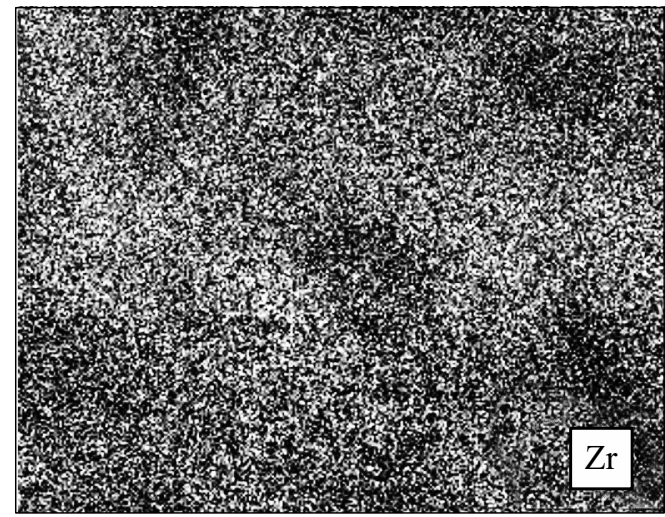

$d$

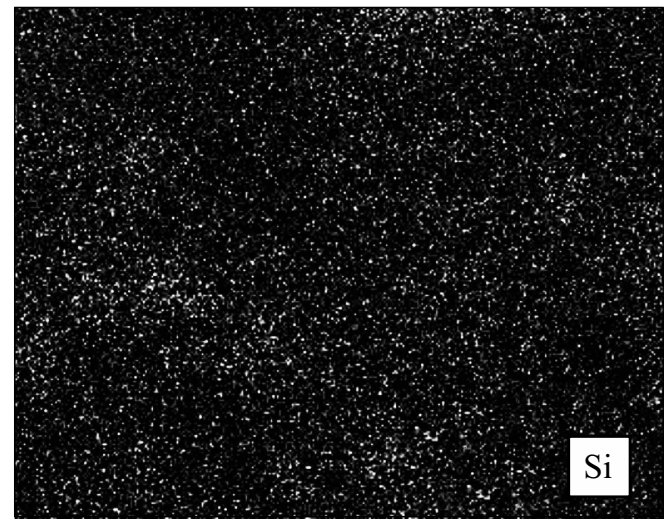

Рис. 8. Характерное СЭМ-изображение участка поверхности скола образца $S 5(a)$ и карты распределения $\mathrm{Zr}(b), \mathrm{Al}(c)$ и $\mathrm{Si}(d)$ по тому же участку поверхности.

во-вторых, различием средних размеров кристаллитов в сравниваемых керамиках.

Увеличение вязкости разрушения $K_{C}$ на $75 \%$ (рис. 4, $a$ ), а также увеличение относительной доли моноклинной фазы в деформированной области материала (рис. 2,6) при введении в ATZ-керамику 5 mol.\% диоксида кремния свидетельствует о повышении эффективности (снижении энергетического порога) $t \rightarrow m$-фазовых превращений диоксида циркония. Это косвенно подтверждается уменьшением средних контактных напряжений под индентором на 16\% при введении в ATZ-керамику диоксида кремния (рис. $5, b)$.

\section{Заключение}

В работе показано, что введение нескольких процентов диоксида кремния в диоксидциркониевую керамику, стабилизированную оксидом кальция $\left(C_{\mathrm{CaO}}=6.5 \mathrm{~mol} . \%\right)$ и упрочненную оксидом алюминия $\left(C_{\mathrm{Al}_{2} \mathrm{O}_{3}}=5.8 \mathrm{~mol} . \%\right)$, вызывает значительное улучшение ее механических свойств. Установлено оптимальное с точки зрения комплекса механических свойств содержание $\mathrm{SiO}_{2}$ в ATZкерамике. Введение 5 mol.\% диоксида кремния приводит к существенному увеличению вязкости разрушения (от 7.05 до $12.4 \mathrm{MPa} \cdot \mathrm{m}^{0.5}$ ) при незначительном снижении твердости (от 12.7 до $10.9 \mathrm{GPa}$ ). Кроме того, при этом наблюдается увеличение прочности на сжатие (от 2.45 до $2.75 \mathrm{GPa}$ ) и пластичности (от 5.3 до 7.3\%). Показано, что улучшение механических свойств вызвано повышением трансформируемости тетрагональной фазы $t-\mathrm{ZrO}_{2}$.

Превышение 5-процентного содержания $\mathrm{SiO}_{2}$ в ATZ-керамике ведет к резкой деградации ее механических свойств, связанной со спонтанным $t \rightarrow m$-фазовым превращением диоксида циркония при охлаждении после спекания. Предполагается, что деградация вызвана взаимодействием $\mathrm{SiO}_{2}$ и $\mathrm{CaO}$ с образованием силиката кальция и соответствующим уменьшением концентрации стабилизатора тетрагональной фазы диоксида циркония.

Анализ рентгеновских дифрактограмм позволил установить, что кремнийсодержащие фазы находятся в аморфном состоянии и в соответствии с данными элементного картирования равномерно распределены по границам зерен.

Таким образом, введение диоксида кремния в ATZ-керамику (стабилизированную оксидом кальция) может быть использовано для улучшения комплекса механических свойств. При этом необходимо соблюдать строгое соотношение концентраций компонентов, поскольку 
превышение порогового значения концентрации $\mathrm{SiO}_{2}$ вызывает резкое ухудшение механических свойств ATZкерамики.

\section{Благодарности}

Результаты были получены с использованием оснащения Центра коллективного пользования научным оборудованием ТГУ им. Г.Р. Державина. Авторы благодарят Виктора Коренкова, Владимира Васюкова и Александра Жигачева за помощь в регистрации рамановских спектров, $\sigma-\varepsilon$-диаграмм и получении СЭМ-изображений соответственно.

\section{Финансирование работы}

Исследование выполнено при финансовой поддержке РФФИ в рамках научных проектов № 19-03-00634 и № 18-29-17047.

\section{Конфликт интересов}

Авторы заявляют, что у них нет конфликта интересов.

\section{Список литературы}

[1] R.H.J. Hannink, P.M. Kelly. J. Am. Ceram. Soc., 83, 461 (2000). DOI: org/10.1111/j.1151-2916.2000.tb01221.x

[2] R.C. Garvie, R.H.J. Hannink, R.T. Pascoe. Nature, 258, 703 (1975).

[3] B. Basu, K. Balani. Advanced structural ceramics. Hoboken: (Wiley, 2011) 504 p.

[4] G. Soon, B. Pingguan-Murphy, K.W. Lai, S.A. Akbar. Ceram. Int., 4, 12543 (2016). DOI: 10.1016/j.ceramint.2016.05.077

[5] A.G. Evans. J. Am. Ceram. Soc., 73, 187 (1990).

[6] M.H. Ghaemi, S. Reichert, A. Krupa, A. Zykova, K. Lobach, S. Sayenko, Y. Svitlychnyi. Ceram. Int., 43, 9746 (2017). DOI: org/10.1016/j.ceramint.2017.04.150

[7] A. Maji, G. Choubey. Mater. Today Proceed., 5, 7457 (2018). DOI: 10.1016/j.matpr.2017.11.417

[8] M.C. Aragón-Duarte, A. Nevarez-Rascón, H.E. EsparzaPonce, M.M. Nevarez-Rascón, R.P. Talamantes, C. Ornelas, J. Mendez-Nonell, J. González-Hernández, M.J. Yacamán, A. Hurtado-Macías. Ceram. Int., 43, 3931 (2017). DOI: org/10.1016/j.ceramint.2016.12.033

[9] S. Sequeira, M.H. Fernandes, N. Neves, M.M. Almeida. Ceram. Int., 43, 693 (2017). DOI: org/10.1016/j.ceramint.2016.09.216

[10] J.-K. Lee, M.J. Kim, E.G. Lee. J. Mater. Sci. Lett., 21, 259 (2002). DOI: org/10.1023/A:1014737614591

[11] F. Zhang, L.F. Lin, E.Z. Wang. Ceram. Int., 41, 2417 (2015). DOI: org/10.1016/j.ceramint.2015.06.081

[12] S. Roya, J. Gibmeiera, K.G. Schell, E.C. Bucharsky, K.A. Weidenmann, A. Wanner, M.J. Hoffmann. Mater. Sci. Eng. A., 753, 247 (2019). DOI: 10.1016/j.msea.2019.03.049

[13] V.R. Khrustov, V.V. Ivanov, S.V. Zayats, A.S. Kaygorodov, S.N. Paranin, S.O. Cholakh. Inorg. Mater. App. Res., 5, 482 (2014). DOI: 10.1134/S2075113314050098

[14] A. Smirnov, J.F. Bartolome, H.D. Kurland, J. Grabow, F.A. Muller. J. Am. Ceram. Soc., 99, 3205 (2016). DOI: $10.1111 /$ jace. 14460
[15] V. Verma, B.V.M. Kumar. Mater. Today Proceed., 4, 3062 (2017). DOI: org/10.1016/j.matpr.2017.02.189

[16] J. Fan, T. Lin, F. Hu, Y. Yu, M. Ibrahim, R. Zheng, Sh. Huang, J. Ma. Ceram. Int., 43, 3647 (2017). DOI: org/10.1016/j.ceramint.2016.11.204

[17] M. Michálek, J. Sedláček, M. Parchoviansky, M. Michálková, D. Galusek. Ceram. Int., 40, 1289 (2014). DOI: org/10.1016/j.ceramint.2013.07.008

[18] А.А. Дмитриевский, А.О. Жигачев, Д.Г. Жигачева, А.И. Тюрин. ЖТФ, 89, 107 (2019). [А.А. Dmitrievskii, A.O. Zhigachev, D.G. Zhigacheva, A.I. Tyurin. Tech. Phys., 64, 103 (2019)] DOI: 10.1134/S1063784219010092

[19] S.E. Porozova, V.B. Kulmetyeva. Inorg. Mater. App. Res., 5, 420 (2014). DOI: org/10.1134/S2075113314040406

[20] I. Danilenko, G. Lasko, I. Brykhanova, V. Burkhovetski, L. Ahkhozov. Nanoscale Res. Lett., 12, 125 (2017). DOI: org/10.1186/s11671-017-1901-7

[21] A.O. Zhigachev, V.V. Rodaev, A.V. Umrikhin, Yu.I. Golovin. Ceram. Int., 45, 627 (2019). DOI: org/10.1016/j.ceramint.2018.09.220

[22] M.L. Mecartney. J. Am. Ceram. Soc., 70, 54 (1987). DOI: org/10.1111/j.1151-2916.1987.tb04853.x

[23] L. Gremillard, J. Chevalier, T. Epicier, G. Fantozzi. J. Am. Ceram. Soc., 85, 401 (2002). DOI: 10.1111/j.1151-2916.2002.tb00103.x

[24] J. Binner, K. Annapoorani, A. Paul, I. Santacruz. J. Eur. Ceram. Soc., 28, 973 (2008). DOI: 10.1016/j.jeurceramsoc.2007.09.002

[25] D. Galusek, K. Ghillányová, J. Sedláček, J. Kozankova. J. Eur. Ceram. Soc., 32, 1965 (2012). DOI: $10.1016 /$ j.jeurceramsoc.2011.11.038

[26] А.А. Дмитриевский, А.И. Тюрин, А.О. Жигачев, Д.Г. Гусева, П.Н. Овчинников. Письма ЖТФ, 44, 25 (2018). [A.A. Dmitrievskii, A.I. Tyurin, A.O. Zhigachev, D.G. Guseva, P.N. Ovchinnikov. Tech. Phys. Lett., 44, 141 (2018).] DOI: $10.1134 / \mathrm{S} 1063785018020219$

[27] A. Moradkhani, H. Baharvandi. Eng. Fract. Mech., 191, 446 (2018). DOI: org/10.1016/j.engfracmech.2017.12.033

[28] Ю.И. Головин. ФТТ, 50, 2113 (2008). [Y.I. Golovin. Phys. Solid State., 50, 2205 (2008).] DOI: org/10.1134/S1063783408120019

[29] M. Arciniegas, Y. Gaillard, J. Pena, J.M. Manero, F.J. Gil. Intermetallics., 17, 784 (2009). DOI: $10.1016 /$ j.intermet.2009.03.020

[30] D.R. Clarke, F. Adar. J. Am. Ceram. Soc., 65, 284 (1982). DOI: org/10.1111/j.1151-2916.1982.tb10445.x

[31] A.M. Limarga, J. Iveland, M. Gentleman, D.M. Lipkin, D.R. Clarke. Acta Mater., 59, 1162 (2011). DOI: $10.1016 /$ j.actamat.2010.10.049

[32] L. Kurpaska, J. Jasinski, E. Wyszkowska, K. NowakowskaLangier, M. Sitarz. Spectrochim. Acta A., 195, 184 (2018). DOI: org/10.1016/j.saa.2018.01.074

[33] C.M. Ramos, P.F. Cesar, R.F.L. Mondelli, A.Sh. Tabata, J.d.S. Santos, A.F.S. Borges. J. Prosth. Dent., 112, 886 (2014). DOI: https://doi.org/10.1016/j.prosdent.2014.02.009

[34] A. Nevarez-Rascon, A. Aguilar-Elguezabal, E. Orrantia, M.H. Bocanegra-Bernal. Int. J. Refract. Met. Hard. Mater., 27, 962 (2009). DOI: 10.1016/j.jjrmhm.2009.06.001

[35] Z. Fan, Y. Zhao, Q. Tan, N. Mo, M.-X. Zhang, M. Lu, H. Huang. Acta Mater., 170, 24 (2019). DOI: $10.1016 /$ j.actamat.2019.03.020

[36] I.A. Aksay, J.A. Pask. Science, 11, 69 (1974). DOI: $10.1126 /$ science. 183.4120 .69

[37] S. Kwon, W.Y. Kim, P. Hudon, I.-H. Jung. J. Eur. Ceram. Soc., 37, 1095 (2017). DOI: 10.1016/j.jeurceramsoc.2016.10.011 